\title{
Inhibitory action of the toxic compounds present in lignocellulosic hydrolysates on xylose-to-xylitol bioconversion by Candida guilliermondii
}

\author{
R. S. Pereira ${ }^{1}$, S. I. Mussatto ${ }^{2}$, and I. C. Roberto ${ }^{1}$ \\ ${ }^{1}$ Department of Biotechnology, Engineering College of Lorena, University of São Paulo, Estrada Municipal do Campinho s/n, \\ 12602-810, Lorena/ SP, Brazil \\ ${ }^{2}$ IBB - Institute for Biotechnology and Bioengineering, Centre of Biological Engineering, University of Minho, Campus de \\ Gualtar, 4710-057, Braga, Portugal.
}

Lignocellulosic materials represent an abundant and inexpensive source of sugars which can be microbiologically converted to industrial products. However, hydrolysis of lignocellulosic materials for sugars recovery always goes together with the formation of by-products that inhibit the fermentation process. Such by-products include acetic acid, phenolic compounds such as syringaldehyde, ferulic acid, $p$-hydroxybenzoic acid, and vanillic acid, among others. These toxic compounds can stress fermentative organisms to a point beyond which the efficient utilization of sugars is reduced and product formation decreases. The inhibitory concentration of these compounds vary to each microorganism and the knowledge about the maximum tolerable concentration and how to minimize their effects is of great importance to attain efficient fermentative processes. Regarding the industrial products that can be generated from lignocellulose-derived sugars, xylitol has received much attention, since it presents particular physic-chemical properties, which permit its use either in foods as a sweetener with anticariogenic property, or in medicines as a sugar substitute for the treatment of diabetes and erythrocytic glucose-6-phosphate dehydrogenase deficiency. In this study, the inhibitory action of acetic acid, ferulic acid and syringaldehyde on metabolism of Candida guilliermondii yeast during the xylose-to-xylitol bioconversion was evaluated. The concentration values evaluated for each toxic compound were based on values usually found in lignocellulosic hydrolysates.

For a good understanding of the individual effect of each toxic compound, the assays were performed in semi-defined medium containing (g/l): xylose (85.0), glucose (15.0), $\left(\mathrm{NH}_{4}\right)_{2} \mathrm{SO}_{4}(3.0), \mathrm{CaCl}_{2} \times 2 \mathrm{H}_{2} \mathrm{O}(0.1), \mathrm{KH}_{2} \mathrm{PO}_{4}(21.7), \mathrm{K}_{2} \mathrm{HPO}_{4}$ (6.9), and $20 \%(\mathrm{v} / \mathrm{v})$ rice bran extract; supplemented or not with acetic acid $(0.8$ to $2.6 \mathrm{~g} / \mathrm{l})$, ferulic acid $(0.2$ to $0.6 \mathrm{~g} / \mathrm{l})$ or syringaldehyde ( 0.3 to $0.8 \mathrm{~g} / \mathrm{l})$. The experiments were performed in $250-\mathrm{ml}$ Erlenmeyer flasks containing $100 \mathrm{ml}$ of fermentation medium inoculated with $3 \mathrm{~g} / \mathrm{l}$ cells. The flasks were incubated at $30^{\circ} \mathrm{C}, 200 \mathrm{rpm}$, for $96 \mathrm{~h}$. During the fermentation, samples were withdrawn each $24 \mathrm{~h}$ for determination of the xylose, glucose, xylitol, and cells concentration, as well as the $\mathrm{pH}$ variations.

The yeast cell growth was affected by all the evaluated acetic acid concentrations, while the xylose consumption and xylitol formation were only affected in the presence of the highest acid concentration $(2.6 \mathrm{~g} / \mathrm{l})$. In fact, the presence of this acetic acid concentration in the fermentation medium reduced the cell growth, xylose consumption and xylitol production in 30,13 and $18 \%$, respectively, when compared to the assay without the acid addition. Cell growth was also reduced in the presence of all the evaluated ferulic acid concentrations, being observed a decrease of $30 \%$ in the microorganism growth when the fermentation medium was supplemented with the maximum ferulic acid concentration $(0.6 \mathrm{~g} / \mathrm{l})$. On the other hand, xylose consumption was not affected by the ferulic acid presence in any evaluated concentration, and the xylitol production was only slightly reduced $(15 \%)$ when in the presence of $0.6 \mathrm{~g} / 1$ ferulic acid. Syringaldehyde concentrations higher than $0.3 \mathrm{~g} / \mathrm{l}$ affected the yeast cell growth, being observed a reduction of $36 \%$ on biomass formation when the fermentation medium was supplemented with $0.5 \mathrm{or} 0.8 \mathrm{~g} / \mathrm{l}$ of this toxic compound. However, the xylose consumption and xylitol formation were not affected by any of the tested concentrations of this compound.

It was thus concluded that acetic acid, ferulic acid, and syringaldehyde are compounds that may affect the Candida guilliermondii metabolism (mainly the cell growth) during the xylose bioconversion to xylitol, being their toxic effect dependent of the concentration present in the medium. Such results are of interest and reveal that it is not necessary a complete removal of toxic compounds from the fermentation medium to obtain an efficient xylose conversion to xylitol by Candida guilliermondii. Acknowledgements: CAPES, FAPESP and CNPq, Brazil.

Keywords xylose; xylitol; Candida guilliermondii; acetic acid; ferulic acid; syringaldehyde; toxic compounds 